\title{
Strategien zur Erhöhung der Organspenderzahlen
}

\author{
Es besteht Konsens, dass Massnahmen zur Erhöhung der Zahl von Organspendern \\ nötig sind. Aber welche? Förderung der Lebendspende, finanzielle Abgeltung, Re- \\ ziprozitätsmodell, gesetzliche Bestimmungen? Bei jedem Ansatz gibt es Pro und \\ Contra.
}

Julius Weiss, Marc Marthaler, Franz F. Immer

Swisstransplant, Schweizerische Nationale Stiftung für Organspende und Transplantation

Korrespondenz:

PD Dr. med. Franz F. Immer FMH Herz- und thorakale Gefässchirurgie

Direktor Swisstransplant

Laupenstrasse 37

CH-3008 Bern

Tel. 0313808133

Fax 0313808132

franz.immer@

swisstransplant.org

www.swisstransplant.org
Das Angebot von Organen für die Transplantation ist seit Jahren zu gering, und die Nachfrage steigt sogar weiterhin stetig an - dies mit gravierenden Folgen für Menschen, die ein Organ benötigen und sich auf immer länger werdenden Wartelisten wiederfinden. Es besteht deshalb ein weitgehender Konsens, dass wirkungsvolle Massnahmen zur Erhöhung der Spenderzahlen dringend nötig sind [1-3]. Wenig Einigkeit herrscht allerdings darüber, mit welchen Mitteln dieses Ziel am besten erreicht werden kann. Verschiedene Lösungsansätze stehen zur Diskussion - wobei allen Beteiligten klar ist, dass nicht länger debattiert, sondern entschieden gehandelt werden muss.

Tatsächlich ist es nicht so, dass die in die Organtransplantation involvierten Kreise bisher untätig waren. Verschiedenste Massnahmen wurden und werden ergriffen, um die Spenderzahlen zu erhöhen: Informationskampagnen für die Bevölkerung, eine verbesserte Spenderdetektion in den Spitälern, die Wiederaufnahme des Non-Heart-Beating-Donor-Programms in der Schweiz, um nur einige zu nennen. Auch wenn jede dieser Massnahmen einen wertvollen Beitrag zur Verbesserung der Situation leistet, so ist der grosse Durchbruch bisher leider nicht gelungen. Zwar ist in der Schweiz unlängst ein leichter Aufwärtstrend bei den Spenderzahlen zu verzeichnen; dieser startet jedoch auf vergleichsweise tiefem Niveau und kann daher nicht über den akuten Mangel an Organen hinwegtrösten.

Die Gesellschaft ist es ihren schwächsten Mitgliedern - den Kranken, die dringend ein Organ benötigen - schuldig, die Lösung des Problems des Organmangels nicht länger aufzuschieben. Denn aufschieben bedeutet im schlimmsten Fall, Menschen sterben zu lassen, die leben könnten, gäbe es mehr Organspender. Alle sind also aufgefordert, ihren Beitrag zu leisten. Die Lösungsmöglichkeiten sind bekannt und ebenso, dass Nichtstun keine Option ist. Als Entscheidungshilfe, sei es für die persönliche Entscheidung pro oder contra Organspende, sei es für den ebenso notwendigen politischen Entschluss, wie gegen den Organmangel vorgegangen werden soll, wird des- halb hier eine Übersicht der Handlungsoptionen präsentiert: die Förderung der Lebendspende, die finanzielle Abgeltung der Organspende, das Reziprozitätsmodell sowie die Förderung der Organspende mittels gesetzlicher Bestimmungen.

\section{Förderung der Lebendspende}

\section{Grundgedanke}

Bei Niere, Leber, Dünndarm und Lunge ist eine Lebendspende möglich. Die Warteliste für die meisten Organe ist so lang, dass sie mit Leichenspendern alleine nicht reduziert werden kann, was gravierende Folgen sowohl für die Lebensqualität der Patienten auf der Warteliste hat als auch beträchtliche Kosten verursacht, die nicht entstehen würden, stünden mehr Spenderorgane zur Verfügung. Würden sich mehr Leute für eine Lebendspende entscheiden, so könnten diese negativen Konsequenzen des Organmangels wenigstens teilweise abgeschwächt werden.

\section{Pro}

Durch eine Lebendspende kann die Wartezeit für ein Organ verkürzt und der Zeitpunkt der Transplantation optimal geplant werden. Dies führt dazu, dass die Resultate nach einer Lebendspende im Vergleich mit einer Leichenspende im Durchschnitt deutlich besser sind [4]. Bei einer frühzeitigen Transplantation, wie sie bei Lebendnierenspenden (der häufigsten Art der Lebendspende) möglich ist, besteht zudem der Vorteil, dass die Transplantation, verglichen mit der Dialyse, sowohl kostensparend als auch mit einer signifikanten Verbesserung der Lebensqualität verbunden ist.

\section{Contra}

Zwar basiert die Lebendspende auf dem Grundsatz der Freiwilligkeit (Transplantationsgesetz, Abs. 3), trotzdem kann bei einer Lebendspende zugunsten von Familienangehörigen nicht ausgeschlossen werden, dass die Spender eine besondere moralische Verpflichtung verspüren oder gar ein impliziter Druck vonseiten der Familie zur Lebendspende ausgeübt wird. 
Ein anderer Nachteil besteht darin, dass eine Lebendspende für den Spender ein - wenn auch geringes - Risiko mit sich bringt. Des weiteren ist die Frage ungeklärt, was geschieht, wenn Lebendspender zu einem späteren Zeitpunkt selber ein Organ brauchen. Bisher ist nicht vorgesehen, dass bei der Organzuteilung berücksichtigt wird, ob jemand Lebendspender ist, wie es in diesem Fall gerechtfertigt schiene. Ausserhalb des familiären Umfelds kann überdies kaum erwartet werden, dass viele Menschen das Risiko einer altruistischen Spende auf sich nehmen, bei der sie keinen persönlichen Bezug zum Empfänger haben.

\section{Finanzielle Abgeltung der Organspende}

Obwohl die Unentgeltlichkeit der Organspende in der Schweiz im Bundesgesetz über die Transplantation von Organen, Geweben und Zellen (Transplantationsgesetz, Art. 6) verankert und der Handel mit Organen verboten ist (Art. 7), soll die finanzielle Abgeltung der Organspende hier trotzdem diskutiert werden, da sie als Option zur Lösung des Problems des Organmangels immer wieder ins Gespräch gebracht wird.

\section{Grundgedanke}

Wo eine Nachfrage besteht, wird ein Markt mit entsprechendem Angebot entstehen. Da es zu wenig Organspender gibt, die ihre Organe unentgeltlich zur Verfügung stellen, können finanzielle Anreize eine Motivation zum Organspenden sein.

Pro

Die Welt, in der wir leben, ist nicht perfekt; denn in einer solchen würde es genügend Spenderorgane geben. Nun besteht aber ein Mangel an Organspenden, der dazu führt, dass in gewissen Ländern mit Organen gehandelt wird [5]. Dies ist zwar in fast allen Ländern illegal, aber damit lässt sich - wie bei anderen illegalen Märkten - der Handel nicht aus der Welt schaffen. Eine der Folgen davon ist, dass weder diejenigen, die mehr oder minder freiwillig ihre Organe geben, noch die Organempfänger in erster Linie davon profitieren, sondern Mittelsmänner, die den illegalen Handel organisieren und betreiben. Eine nationale oder internationale Regulierung des Organhandels scheint somit die einzige Möglichkeit zu sein, die negativen Konsequenzen zu minimieren, die ein unkontrollierter Markt sowohl für Anbieter als auch für Abnehmer mit sich bringt [6].

\section{Contra}

Es bestehen Bedenken, dass bei einer Bezahlung der Organspende die Motivation, Organe unentgeltlich zur Verfügung zu stellen, abnehmen würde, und somit insgesamt keine Erhöhung der Spenderzahlen erreicht wird [7]. Zudem ist es naheliegend anzunehmen, dass primär sozial schlechter gestellte Menschen ihre Organe gegen Bezahlung anbieten würden. Dies müsste als Ausnutzung einer Notlage gewertet werden, was moralisch höchst bedenklich ist und deshalb keine Option darstellt [8].

\section{Reziprozitätsmodell}

\section{Grundgedanke}

Organtransplantationen kann es nur geben, wenn es auch Organspender gibt - wie man nur etwas schenken kann, wenn es Geschenke gibt. Dies bedeutet, dass jeder, der nicht ausschliessen kann, dass er ein Organ annehmen würde, falls er eines brauchen würde, sich daher konsequenterweise bereit erklären müsste, auch Organspender zu sein [9]. Es ist nicht einsichtig, weshalb Leute, die es ablehnen, ihre Organe zu spenden, ein Anrecht auf ein Spenderorgan haben sollten; denn wenn sich alle so verhalten würden, stünden gar keine Organe für eine Transplantation zur Verfügung.

\section{Nichtstun ist keine Option}

\section{Pro}

Das Risiko, im Laufe des Lebens einmal auf ein Spenderorgan angewiesen zu sein, ist sieben Mal grösser als die Chance, dass man selbst zum Spender wird. So betrachtet weist die Bereitschaft zur Organspende gewisse Ähnlichkeiten mit einer Versicherung auf. Es ist daher widersinnig, dass Leute, die nicht bereit sind, einen bescheidenen Beitrag zu leisten, im Bedarfsfall trotzdem in vollem Umfang von den Vorteilen der Organtransplantation profitieren sollten [7]. Darüber hinaus kann die Möglichkeit von Trittbrettfahrern dazu führen, dass das System als unfair betrachtet wird und damit die Motivation, sich für eine Organspende zu entscheiden, untergraben wird.

\section{Contra}

Die Bevorzugung von erklärten Spendern gegenüber Nichtspendern widerspricht dem Grundsatz, dass bei der Zuteilung von Organen niemand diskriminiert werden darf (Transplantationsgesetz, Art. 17). Zudem würde es bedeuten, dass nicht-medizinische Kriterien bei der Organzuteilung berücksichtigt werden, worauf bisher mit guten Gründen verzichtet wurde.

Unklar bleibt, wie ein solches Reziprozitätsmodell umgesetzt werden sollte, damit die Gegenseitigkeit tatsächlich als ein faires Kriterium bei der Organzuteilung berücksichtigt werden könnte: Wie kann ausgeschlossen werden, dass Leute erst eine Spendekarte ausfüllen, wenn sie wissen, dass sie eine Transplantation benötigen (und damit den Gedanken der Gegenseitigkeit unterlaufen)? 


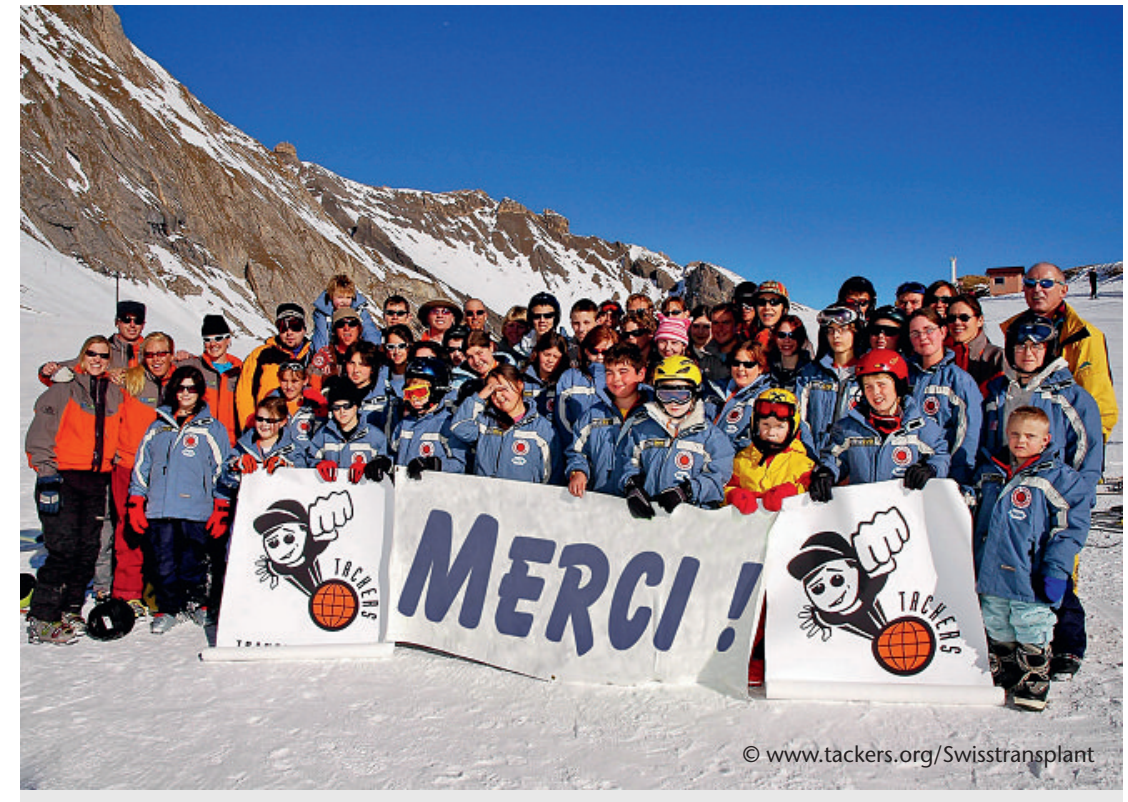

Die Organspende - ein geschenktes neues Leben: Teilnehmer des internationalen Winterlagers für transplantierte Kinder «TACKERS» (Transplant Adventure Camps for Kids).

\section{Förderung der Organspende mittels gesetzlicher Bestimmungen}

\section{Grundgedanke}

Es ist im Interesse der Menschen, die ein Organ benötigen, dass es möglichst viele Spender gibt. Ausserdem ist eine Transplantation, verglichen mit anderen Therapieformen, in den meisten Fällen längerfristig kostensparend, weshalb die Förderung der Organspende in dieser Hinsicht auch im Sinne der Allgemeinheit ist. Es scheint deshalb einleuchtend, dass der Gesetzgeber Bestimmungen erlässt, die dazu führen, dass möglichst viele Leute einer Organspende zustimmen.

\section{Widerspruchslösung anstelle Zustimmungslösung}

Eine Option wäre, anstelle der zur Zeit geltenden Zustimmungslösung - bei der eine Organentnahme im Todesfall nur möglich ist, wenn der Verstorbene (beziehungsweise dessen Angehörige) sein explizites Einverständnis zur Organspende vorgängig gegeben hat - die Widerspruchslösung im Transplantationsgesetz zu verankern. Die Widerspruchslösung sieht vor, dass jeder, der nicht ausdrücklich festgehalten hat, dass postmortal keine Organe entnommen werden dürfen, als Organspender in Frage kommt.

\section{Pro}

In vielen Ländern spricht sich eine Mehrheit der Bevölkerung bei Befragungen für die Organspende aus [10]. Es kann daher davon ausgegangen werden, dass es sich bei der Organspende um sozial erwünschtes Verhalten handelt. Dies sollte der Gesetzgeber dahingehend berücksichtigen, als dass die Zustimmung zur Organspende als gegeben angenommen wird und die
Bestimmungen so lauten, dass diejenigen, die sich gegen eine Organspende entscheiden, dies aktiv zum Ausdruck bringen müssen, und nicht diejenigen, die sich für die Spende entscheiden.

\section{Contra}

Es ist fraglich, wie gross der positive Einfluss der Widerspruchslösung auf die Spenderzahlen ist. Eindeutig scheint nur, dass es naiv wäre zu glauben, alleine mit der Einführung der Widerspruchslösung würden die Spenderzahlen automatisch wesentlich steigen [11]. Ausserdem ist klärungsbedürftig, welche Auswirkungen die Regelung auf Kinder und urteilsunfähige Personen hat.

\section{Pflicht zur postmortalen Organspende}

In einem Kontext wie der Organspende, in dem der Freiwilligkeit und dem Altruismus gemeinhin ein grosses Gewicht beigemessen werden, mag es im ersten Moment befremdlich anmuten, wenn von einer Pflicht die Rede ist. Betrachtet man die Organspende jedoch als eine Form der Nothilfe - nämlich jemandem indirekt das Leben zu retten -, so erscheint der Gedanke, dass man vom Gesetzgeber dazu verpflichtet werden könnte (in Analogie zur Pflicht, Nothilfe zu leisten bei Menschen, die sich in unmittelbarer Lebensgefahr befinden) nicht mehr so abwegig [12].

\section{Pro}

Es gibt verschiedene Bürgerpflichten, welche die Freiheit des Einzelnen einschränken; neben der Nothilfe beispielsweise die Wehrpflicht oder die Pflicht, Steuern zu zahlen. Berücksichtigt man, dass mit einer Organtransplantation das Leben eines Patienten gerettet werden kann, so lässt sich, im Sinne einer Güterabwägung, die Einschränkung der Wahlfreiheit bezüglich der postmortalen Organspende rechtfertigen. Das Kriterium der Zumutbarkeit, das den Umfang der Nothilfe einschränkt, würde einer Pflicht zur Lebendspende (bei welcher der Spender ein gewisses gesundheitliches Risiko trägt) widersprechen. Bei der postmortalen Spende erwachsen dem Spender jedoch keine Risiken, die im Vergleich zum Nutzen, den die Empfänger von einer Transplantation haben, als unzumutbar betrachtet werden können [13].

\section{Contra}

Im Falle einer Ablehnung der Organspende ist der zu Lebzeiten geäusserte Wille einer verstorbenen Person (beziehungsweise der Wille der Angehörigen) höher zu gewichten als das Interesse derjenigen, die ein Organ benötigen [14]. Eine postmortale Organspendepflicht kann im Widerspruch zu religiösen oder weltanschaulichen Überzeugungen stehen und stellt damit einen zu grossen Eingriff in die persönliche Freiheit dar, weshalb eine solche Lösung prinzipiell abzulehnen ist. 


\section{Fazit}

Die hier vorgestellten Strategien zur Erhöhung der Organspenderzahlen haben - wie nicht anders zu erwarten - allesamt ihre Vor- und Nachteile. Es gibt also keine Strategie, die das Problem des Spendermangels lösen würde, ohne dass gewisse unerwünschte Konsequenzen in Kauf genommen werden müssen. Allerdings kann dies kein Grund sein, keine Entscheidung zu treffen. Denn damit wird verhindert, dass Men-

\section{«Welche Nachteile können wir zugunsten einer Verbesserung der Situation akzeptieren?»}

schen geholfen werden kann, die ohne ein Spenderorgan entweder unter einer wesentlich verminderten Lebensqualität leiden oder die aufgrund des Organmangels gar auf der Warteliste versterben. Die Frage, die zu beantworten ist, lautet demnach: «Welche Nachteile können wir zugunsten einer Verbesserung der Situation akzeptieren?» Und die Antwort gilt es umzusetzen.

\section{Literatur}

1 Nett PC, Stüssi G, Weber M, Seebach JD. Transplantationsmedizin: Strategien gegen den Organmangel. Schweiz Med Forum. 2003;24(11):559-68

2 Abouna GM. Organ shortage crisis: problems and possible solutions. Transplant Proc. 2008;40:34-8.

3 Flückiger A (Hrsg.). Emouvoir et persuader pour promouvoir le don d'organes? - L'efficacité entre éthique et droit. Genève: Schulthess; 2010.
4 Thiel GT. Nieren-Lebendspende im Grenzgebiet von Klinik, Ethik und Recht. Kodex für den Umgang mit Lebendspendern. In: Breyer F, Engelhard M (Hrsg.). Anreize zur Organspende. Graue Reihe, Nr. 39. Europäische Akademie zur Erforschung von Folgen wissenschaftlich-technischer Entwicklungen Bad Neuenahr-Ahrweiler GmbH; 2006; S. 143-170.

5 Steering Committee of the Istanbul Summit. Organ trafficking and transplant tourism and commercialism: the Declaration of Istanbul. Lancet. 2008;372:5-6.

6 Friedman EA, Friedman AL. Payment for donor kidneys: Pros and Cons. Kidney Int. 2006;69:960-2.

7 Illies C, Weber F. Organ trade versus reciprocity model. An ethical evaluation. Dtsch Med Wochenschr. 2004;129:271-5.

8 Budiani-Saberi DA, Delmonico FL. Organ trafficking and transplant tourism: a commentary on the global realities. Am J Transplant. 2008;8:925-9.

9 Lavee J, Ashkenazi T, Gurman G, Steinberg D. A new law for allocation of donor organs in Israel. Lancet. 2010;375:1131-3.

10 European Commission. Special Eurobarometer 272D «Europeans and organ donation». 2007.

11 Rithalia A, McDaid C, Suekarran S, Myers L, Sowden A. Impact of presumed consent for organ donation on donation rates: a systematic review. BMJ. 2009;338:a3162.

12 Wille S. Sozialpflicht zur Organspende? In: Breyer F, Engelhard M (Hrsg.). Anreize zur Organspende. Graue Reihe Nr. 39. Europäische Akademie zur Erforschung von Folgen wissenschaftlich technischer Entwicklungen Bad Neuenahr-Ahrweiler $\mathrm{GmbH}_{\text {; }}$ 2006; S. 7-26.

13 Spital A, Taylor JS. Routine recovery of cadaveric organs for transplantation: consistent, fair, and life-saving. Clin J Am Soc Nephrol. 2007;2:300-3.

14 Hamer CL, Rivlin MM. A stronger policy of organ retrieval from cadaveric donors: some ethical considerations. J Med Ethics. 2003;29:196-200. 